\title{
The Effect of Group Identity on Distributive Choice: Social Preference or Heuristic? और
}

\author{
Francesco Guala ${ }^{\mathrm{a}}$, Antonio Filippin ${ }^{\mathrm{a}, \mathrm{b}}$ \\ ${ }^{a}$ University of Milan, Department of Economics, Via Conservatorio 7, 20122 Milano, Italy \\ ${ }^{b}$ Institute for the Study of Labor (IZA), Schaumburg-Lippe-Str. 5-9, 53113 Bonn, Germany
}

\begin{abstract}
Group identity can influence significantly people's attitudes toward monetary allocations. In this paper we reassess the representation of group identity using social preference models. First, we show that the influence of group identity varies unsystematically across different types of mini-dictator games and cannot be described using a well-behaved preference function. Second, we demonstrate that the effect is not robust to slightly increasing the complexity of the task, suggesting that group identity is a framing effect that can be easily displaced by alternative decision heuristics.
\end{abstract}

\section{JEL Classifications: C92; D03; D30}

Keywords: Identity, groups, dictator game, social preferences.

\footnotetext{
${ }^{\sqrt{3}}$ Research for this paper was funded by a PUR grant and a Rientro dei Cervelli scholarship. We are grateful to Manuela Raimondi and the experimental laboratory of the University of Parma for logistic support. We would like to thank John Smith and participants to a seminar at Nottingham University, to the Essex Experimental Economics Workshop held in June 2013, and to the London Experimental Workshop held in May 2014 for useful suggestions. We take responsibility for all the remaining errors.

Email addresses: francesco.guala@unimi.it (Francesco Guala), antonio.filippin@unimi.it (Antonio Filippin)
} 


\section{Introduction}

Sociologists and social psychologists have been arguing for decades that group membership is an important determinant of behaviour. Economists are late comers in this area of research, but with the rise of experimental economics and renewed interest in other-regarding preferences, several studies of group behaviour have been recently published in major journals, focusing in particular on the effect of group identity. "Group identity" is usually defined as that part of an individual's self-concept that is derived from the affiliation with a social group. In terms that are familiar to economists, we may represent it as a set of beliefs, preferences, norms, or decision heuristics that are dependent on group membership. Experimental and field data suggest that group identity is generally correlated with pro-social attitudes towards the members of one's group. Group identity for example makes us more altruistic, egalitarian, trusting, trustworthy, and cooperative towards fellow members. Interestingly, it also inhibits punishment directed against free riders and norm violators. The dark side of the picture is that group identity fosters negative attitudes towards the members of other groups. It enhances competition, inequality, and the punishment of individuals who do not belong to the same community. ${ }^{1}$

In spite of the wealth of evidence, there is little agreement on how these phenomena should be explained theoretically. An approach that is currently popular among experimental economists invites to model pro-social motives or attitudes as "social preferences". Models of social preferences incorporate concerns for the welfare, the actions, or the intentions of other players by adding extra parameters in the arguments of utility functions. ${ }^{2}$ The impact of group identity thus may be represented as a change in the values of pro-social parameters, and estimated using standard econometric techniques (e.g. Chen and Li, 2009; Benjamin et al., 2010; Bhatia, 2013).

The social preferences approach however is not the only game in town. According to an alternative tradition (Tajfel, 1982; Bacharach, 2006), group identity is essentially a framing

\footnotetext{
${ }^{1}$ See e.g. Akerlof and Kranton (2000, 2010); Bacharach (2006); Benjamin et al. (2010); Bernhard et al. (2006); Charness et al. (2007); Chen and Li (2009); Cooper and Kagel (2005); Eckel and Grossman (2005); Efferson et al. (2008); Filippin and Guala (2013); Goette et al. (2010); Guala et al. (2013); Güth et al. (2009); Hargreaves Heap and Zizzo (2009); Ruffle and Sosis (2006); Sutter (2009); Sugden (2000); Zizzo (2011).

${ }^{2}$ There are more models of social preferences than can be mentioned here, but see Fehr and Schmidt (2006) for a review of the literature.
} 
device. A "frame" is a body of information that subjects exploit to select a response in an unfamiliar or complex decision environment. In the classic "Asian Disease" experiment of Tversky and Kahneman (1986) for example, the way the options are presented (as "lives lost" vs. "lives saved") is strictly speaking irrelevant, but nonetheless triggers an automatic emotional reaction that subjects rely upon when they make their decisions. In the case of group identity, the collective frame may cue a set of heuristics that prescribe generosity and cooperation with the members of one's group.

Some theories combine elements of these two approaches: according to Akerlof and Kranton $(2000,2010)$, for example, group identity is associated with a set of behavioural rules or norms prescribing specific actions in specific circumstances. Their theory differs from the heuristic account, however, because the effect of non-compliance with the norms is included in the argument of people's utility function. ${ }^{3}$ If group identity is a heuristic, in contrast, it should not change the value of options, but rather induce the decision-maker to use particular "fast and frugal" decision rules to economize effort (Gigerenzer et al., 2000). The main difference between these two approaches concerns the regularity of the identity effect. By adding extra parameters, of course, it is always possible to fit experimental data into a utility function. Economists however seek models that combine simplicity with predictive power: the effect of identity on utility functions should be regular across decision contexts. And if the framing interpretation is correct, we should expect social preference parameters capturing group identity to be fragile to small contextual cues that trigger alternative heuristics.

In this paper we report the results of an experiment designed to investigate the regularity of group identity in the context of distributive choice. Our study attempts to extend the results of previous experiments that combine group identity manipulations with the use of mini-dictator games. We move in two directions: first, we test the robustness of social preference models across different distributive choices. To attain more generality we map a broader range of options than previous experiments, by systematically varying the payoffs of the decision-maker and the recipient in a series of games. Second, we test the regularity of the group identity effect by slightly changing the frame of decision through the addition of

\footnotetext{
${ }^{3}$ For a similar attempt to model the influence of social norms, see Krupka and Weber (2013).
} 
dominated options. Previous studies indicate that enlarging the set of options may not only increase the complexity of the decision task, but also change the framing of decision and affect subjects' choices among dominating options. ${ }^{4}$ Since robustness to different frames is a basic requirement for any preference-based explanation, our experiment provides one way to discriminate between social preference and heuristic accounts of group identity.

As we shall see, our data cast a shadow of doubt on social preference accounts. To begin with, we do not find a systematic effect of group identity across the whole range of minidictator games. The effect of group identity on distributive choice is modest and limited to particular types of games. Then we show that the effect changes radically or disappears when dominated options are added to the decision task. This suggests that group identity is a heuristic tool that is highly dependent on contextual factors and that is easily displaced when it competes for cognitive salience with alternative rules of thumb.

The paper is organized as follows: in Section 2 we illustrate the task that we use to measure attitudes toward monetary allocations. Section 3 is devoted to experimental design and procedures. The experimental results are illustrated and discussed in Section 4, while Section 5 summarises and concludes.

\section{Distributive choice and dictator games}

In this paper we are concerned with the simplest kind of distributive choice, that is, the allocation of resources among two individuals. In a standard dictator game, a subject (the "dictator") is given the opportunity to divide a sum of money between her own account and the account of another subject, called the "recipient" (Kahneman et al., 1986; Forsythe et al., 1994). The recipient cannot accept or reject the offer: her role is entirely passive, and as a consequence her payoff should be irrelevant for a perfectly rational dictator who only cares about her own earnings. Nevertheless, a number of studies demonstrate that many experimental subjects give a strictly positive amount to the recipient. This is usually interpreted as proof that other-regarding motives, social preferences, or social norms moderate the basic selfish tendency to maximize one's own payoffs (see e.g. Camerer, 2003).

\footnotetext{
${ }^{4}$ See e.g. Huber and Puto (1982), Simonson (1989).
} 
Dictator games can be used to study the effect of several factors on distributive choice, including group identity. The study of group identity has its roots in the social psychology literature of the 1960s (Sherif and Sherif, 1961; Tajfel et al., 1971). In most studies group identity is either imported in the laboratory as an exogenous variable - for example, when the experimental subjects are already affiliated with two social groups - or is induced endogenously in the laboratory by means of some "priming" procedure. In a typical experiment subjects are first divided in groups on the basis of a salient category. They are then matched with other subjects that belong either to the same group (ingroup condition) or to another group (outgroup condition). Finally, they are asked to perform an economic task with real or fictional payoffs. Any difference that may emerge between the group conditions and a baseline condition without groups is attributed to group identity.

In a recent experiment Bhatia (2013) has used a "modified" dictator game to study the effect of group identity on the price of giving. While in a standard dictator game every unit of payoff that has been transferred to the recipient costs the dictator one unit, in a "modified" game the cost of giving can be lowered or raised at will. This procedure allows the estimation of well-behaved preference relations in the space of self-other payoffs, satisfying classic principles of consumer choice such as the Generalised Axioms of Revealed Preference (Andreoni and Miller, 2002).

The use of "modified" dictator games however has some limitations. First of all, it is meant to estimate well-behaved indifference curves, as it imposes a negative relative price between payoffs (there is always a strictly positive cost that the dictator has to pay in order to increase the payoff of the recipient). The method could in principle be extended to cover budget constraints with non-negative slope, but at the cost of considerable complications in the experimental instructions. Another concern is that investigating a non-negative price of giving may be very expensive for the experimenter.

Such problems can be overcome using a variant known as the "mini-dictator game", as we do in this paper. In a mini-dictator game the decision-maker is required to choose between a finite set of self/other allocations of monetary payoffs, such as:

$$
\text { Option A:(1000, 1000) Option B: }(800,1100)
$$


The first figure within brackets is the payoff of the dictator, while the second figure corresponds to the payoff of the recipient. By forcing subjects to choose among two options it is possible to map preferences over the broadest range of payoffs. Although classic dictator games offer more options than mini-dictator games, the freedom allowed by the former goes largely unexploited because choices tend to cluster around a few salient allocations.

In the above example, option A offers a perfectly equal allocation while B gives the opportunity to benefit the recipient at a cost for the dictator. It is very simple however to modify the payoffs to cover cases that cannot be easily studied using standard dictator games. In the following games for example giving is inexpensive or even advantageous to the dictator:

$$
\begin{array}{ll}
\text { Option A: }(1000,1000) & \text { Option B: }(1000,1100) \\
\text { Option A: }(1000,1000) & \text { Option B: }(1100,1200)
\end{array}
$$

So overall the mini-dictator task allows a better control over the opportunity set and extends the scope of the dictator game. A third argument in favour of mini-dictator games is that they are less sensitive to demand effects than standard or modified dictator games. It is well known that observed allocations in the dictator game vary significantly depending on whether the task is framed as a "giving" or "taking" decision (Bardsley, 2008; List, 2007). In a mini-dictator game, in contrast, the presentation of the options is neutral and the demand effect is diluted by the heterogeneity of the list of choices and by the randomization of the order in which they are presented.

The number of allocations included in the set of options in each mini-dictator game can be made to vary at will. In most experiments, subjects are offered only two options - a simple design that we adopt in our Baseline and Ingroup conditions. Increasing the number of options however provides opportunities to test the robustness of group identity to small changes in the decision environment. We do this by adding a couple of dominated allocations in our Complex and In-Complex conditions, where subjects face four options in each game. These conditions give the opportunity to test the robustness of group identity to a change in the context of decisions that should in principle be irrelevant for a rational decision-maker. 
The results across our experimental conditions can shed interesting light on the nature of the group identity effect. According to the social preferences account, complexity should not alter the effect of group identity. According to the framing account, instead, in a new context the group identity effect may be partially or totally displaced by an alternative rule of thumb. As we shall see, our data lend support to the latter hypothesis, against the social preference interpretation of group identity.

\section{Experimental design and procedures}

The full list of games used in our experiment can be found in Table 1. The order of the binary choices, as well as the labels of the options (A or B) are assigned randomly. One unit is worth one cent of a euro, so for example, 1,000 units $=10$ euro. The games can be classified into five types, called "zero-sum giving", "expensive giving", "cheap giving", "envy", and "spite" games.

Figure 1 provides an intuitive representation, on the plan of self/other payoff profiles, of the kind of options offered. For the sake of simplicity we have only included some representative games rather than all forty-four games. A solid line represents a mini-dictator game with the two options at each end of the line. The forty-five degrees dotted line traces all perfectly egalitarian allocations. Inequalities in the payoffs are favourable to the dictator above that line; below the dotted line, they are favourable to the recipient. A horizontal solid line means that the payoff of the recipient can be increased or decreased at no cost for the dictator (in this case, not giving may be interpreted as envy). A positive slope means that giving is beneficial both to the recipient and to the dictator (not giving may be evidence of spite)..$^{5}$ If the line is negatively inclined, the dictator faces a trade-off between her own payoff and that of the dictator. The slope of the constraint represents the relative price of giving: if the cost is equal to one (every cent to the recipient costs one cent to the dictator) the slope is orthogonal to the equality line and the game is Zero-sum. A steeper line represents Expensive Giving, while a flatter line stands for Cheap Giving games. In general, moving

\footnotetext{
${ }^{5}$ Notice that in Spite games the slope is less than one (more precisely, 0.5), which means that the dictator can reduce the payoff of the recipient of a given amount by reducing his earnings of an amount equal to half of that amount. We gathered evidence in a pilot experiment that the spiteful option is never chosen when the price is $1: 1$.
} 


\begin{tabular}{c|cc|cc|c}
\hline & \multicolumn{2}{|c|}{ Option A } & \multicolumn{2}{|c|}{ Option $\mathbf{B}$} & \\
Round & $\pi_{d}$ & $\pi_{r}$ & $\pi_{d}$ & $\pi_{r}$ & Type \\
\hline 1 & 800 & 1200 & 800 & 1000 & Envy \\
2 & 1200 & 800 & 1400 & 400 & Cheap Giving \\
3 & 1100 & 500 & 1100 & 900 & Envy \\
4 & 1000 & 600 & 1000 & 1000 & Envy \\
5 & 800 & 1200 & 1200 & 1000 & Expensive Giving \\
6 & 900 & 1100 & 700 & 1300 & Zero-Sum Giving \\
7 & 1600 & 400 & 1200 & 800 & Zero-Sum Giving \\
8 & 1200 & 800 & 1200 & 600 & Envy \\
9 & 800 & 1200 & 600 & 1600 & Cheap Giving \\
10 & 800 & 1200 & 1000 & 800 & Cheap Giving \\
11 & 1500 & 500 & 1100 & 900 & Zero-Sum Giving \\
12 & 1400 & 600 & 1000 & 1000 & Zero-Sum Giving \\
13 & 1200 & 400 & 1200 & 800 & Envy \\
14 & 1200 & 800 & 1200 & 1000 & Envy \\
15 & 900 & 1100 & 1300 & 900 & Expensive Giving \\
16 & 900 & 1100 & 900 & 700 & Envy \\
17 & 800 & 1200 & 600 & 1400 & Zero-Sum Giving \\
18 & 900 & 1100 & 1300 & 700 & Zero-Sum Giving \\
19 & 1100 & 900 & 1300 & 700 & Zero-Sum Giving \\
20 & 1100 & 900 & 1500 & 700 & Expensive Giving \\
21 & 1600 & 600 & 1200 & 800 & Expensive Giving \\
22 & 800 & 1400 & 1000 & 1000 & Cheap Giving \\
23 & 1000 & 1000 & 1400 & 800 & Expensive Giving \\
24 & 1000 & 1000 & 1200 & 1400 & Spite \\
25 & 1000 & 1200 & 1000 & 1000 & Envy \\
26 & 1200 & 800 & 1400 & 1200 & Spite \\
27 & 800 & 1200 & 800 & 1600 & Envy \\
28 & 700 & 1300 & 1100 & 900 & Zero-Sum Giving \\
29 & 800 & 1200 & 800 & 1400 & Envy \\
30 & 1200 & 800 & 1000 & 1000 & Zero-Sum Giving \\
31 & 800 & 1200 & 400 & 1600 & Zero-Sum Giving \\
32 & 1100 & 1300 & 1100 & 900 & Envy \\
33 & 1200 & 600 & 1000 & 1000 & Cheap Giving \\
34 & 1000 & 1000 & 800 & 600 & Spite \\
35 & 1100 & 900 & 1300 & 500 & Cheap Giving \\
36 & 900 & 1100 & 500 & 1500 & Zero-Sum Giving \\
37 & 1100 & 1500 & 900 & 1100 & Spite \\
38 & 1000 & 1400 & 1000 & 1000 & Envy \\
39 & 1400 & 600 & 1200 & 800 & Zero-Sum Giving \\
40 & 1200 & 800 & 1000 & 1200 & Cheap Giving \\
41 & 700 & 1500 & 900 & 1200 & Cheap Giving \\
42 & 900 & 1100 & 900 & 1500 & Envy \\
43 & 900 & 1100 & 1100 & 700 & Cheap Giving \\
44 & 1000 & 800 & 1000 & 1000 & Envy \\
\hline$\pi$ & stan for dictator'spayoff & $\pi_{r}$ for & recipient'spayoff. \\
\hline 3 & &
\end{tabular}

$\pi_{d}$ stands for dictator's payoff, $\pi_{r}$ for recipient's payoff.

Table 1: Our set of mini-dictator games 
upward and to the right increases social welfare, intended as the joint payoff of dictator and recipient.

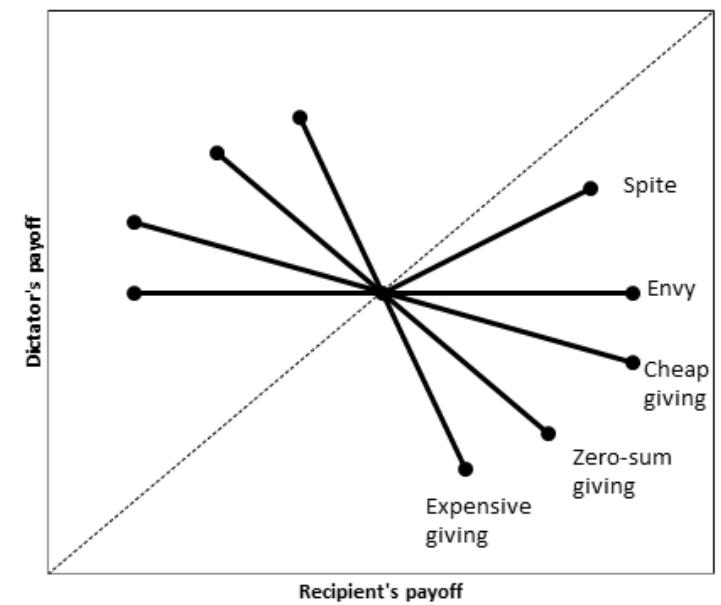

Figure 1: Mini-dictator games in the space of self-other payoffs

The experiment described in this paper is close in spirit to a study recently performed by Chen and $\mathrm{Li}$ (2009), who report the results of twenty-four games in which group identity is manipulated. Of these, however, only five are mini-dictator games with two available options. As we shall see shortly, this leaves a large amount of space unexplored and gives rise to rather idiosyncratic estimates of social preference functions. Because we systematically vary the cost of giving, our experiment is also partly similar to the study of Bhatia (2013) mentioned above. Unlike Bhatia (2013), however, we use mini-dictator games, explore the space of envious and spiteful options, and try to detect the existence of non-well-behaved preference structures (e.g. lexicographic orderings) that cannot be identified using "modified" dictator games a la Andreoni and Miller (2002).

A comparative advantage of our experimental design is that it offers a broader picture of attitudes to distributive choice. The subjects of Chen and Li's experiment for example never face expensive giving, spite, or equality-enhancing envy games (see Figure 2). Such choices, in contrast, are included among the forty-four games of our study. As explained in the next section, such a framework (i) provides an exhaustive map of choices over self-other allocations; (ii) reveals how these choices are affected by group identity in different areas of 
the plane; and (iii) tests the robustness of the group identity effect to small variations in the complexity of the task.

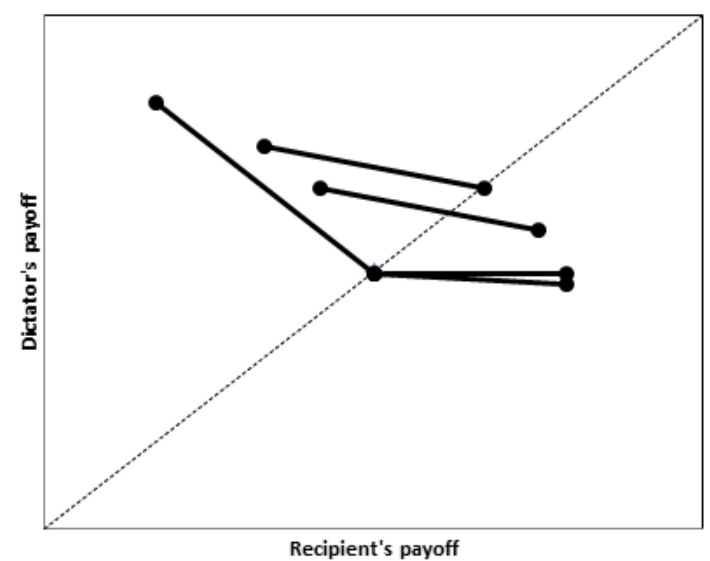

Figure 2: Mini-dictator games of Chen and Li (2009) in the space of self-other payoffs

The design used by Bhatia (2013), in contrast, allows the estimation of preferences when there is a trade-off between self-other allocations, but is unfit to measure the effect of identity in Envy and Spite games. (See Figure 3; for simplicity we have represented on the Cartesian plane only a representative subset of the budget constraints used in this experiment.). Its "modified" dictator games, moreover, cannot guarantee that choices in certain areas of the Cartesian plane are ever observed in the experimental sessions.

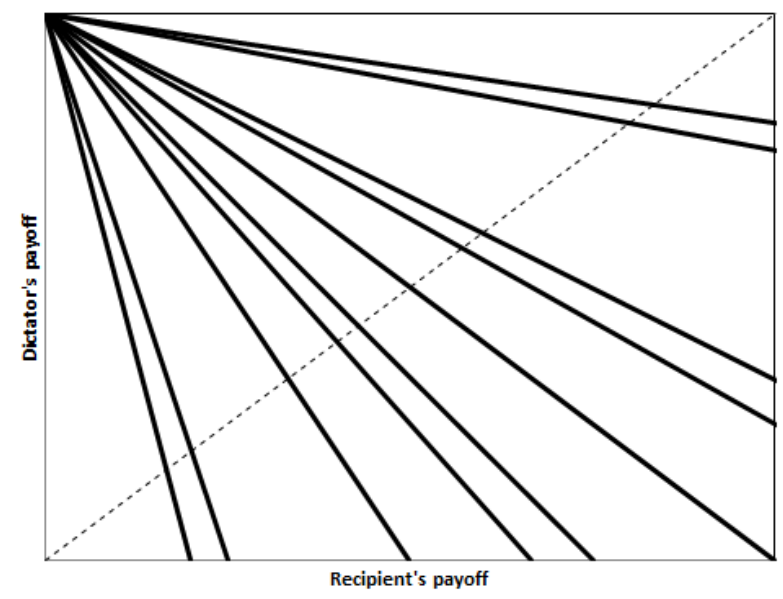

Figure 3: "Modified" dictator games of Bhatia (2013) as budget constraints in the space of self-other payoffs 


\subsection{Design}

Since our experiment is mainly concerned with the effect of identity within groups, we implement only ingroup matchings, and we compare the results with a control condition where subjects have not been assigned to groups. More precisely, the experiment consists of four conditions or treatments in a pure between-subject design: a Baseline condition without group identity; an Ingroup condition with group identity; a Complex condition with a larger set of options; and an In-Complex condition with group identity and a larger set of options. In the Baseline and Ingroup conditions the dictators were asked to choose among two possible allocations. In the Complex and In-Complex conditions in contrast subjects faced four options in each game. Two of these options were identical to those that we offered in the Baseline and Ingroup conditions, and strictly dominated the additional options (in Pareto's sense). The additional options were generated simply by subtracting 200 units from the payoffs of dictator and recipient. For example, when in the other treatments subjects were asked to choose among $(1000,1000)$ and $(800,1100)$, in the two complex conditions they were asked to choose among $(1000,1000),(800,800),(800,1100)$ and $(600,900)$. Notice that in all forty-four choices the second and fourth options are always dominated by the first and third options. In Figure 1 the dominated options could be represented by a parallel solid line located below and to the left of the original choice. Dominated options should never be chosen by rational agents given that they represent the same type of choice as offered by the first and third options. We also doubt that dominated options may be chosen deliberately out of spite, because evidence from a pilot experiment showed that such a choice is never made when subjects are offered binary options with a price of non-giving equal to one.

\subsection{Procedures}

The experiment was articulated in two phases: in the first stage, subjects had to perform a simple memory task; in the second stage they faced the forty-four mini-dictator games described above, in one of the four conditions. The memory task was conducted either individually (Baseline and Complex conditions) or collectively to induce group identity (Ingroup and In-Complex conditions).

In the Baseline and Complex sessions subjects entered the laboratory and were randomly assigned to their computer terminals, separated by partitions. The experimenter read the in- 
structions for the first stage, illustrating the memory task. Each subject received the text of a poem that he/she could study for two minutes. The goal was to memorize and report correctly one verse of the poem. After two minutes the text was withdrawn and the subjects were instructed to write down a verse on a blank sheet of paper provided by the experimenter. Those who reported the line correctly were rewarded with a prize of three euro.

In the Ingroup and In-Complex sessions subjects entered the laboratory and randomly picked a coloured number (Blue or Red) that determined their group affiliation. The members of each group then gathered in separate areas of the laboratory and received the instructions for the first stage of the experiment. The task again was to memorize a poem and to report it correctly on a sheet of paper provided by the experimenter. The number of lines to be memorized was equal to the number of subjects in the group (sixteen). The groups that completed the task successfully received a prize of 48 euro. While the individual payoffs were identical to those of the Baseline and Complex conditions, the task was meant on the one hand to foster coordination to achieve a common goal, and on the other hand to implement the "common fate" condition that is generally considered to be an important determinant of group identity (e.g. Brewer and Kramer, 1986; Zizzo, 2011). Although our techniques are not exactly identical, we make use like Chen and Li (2009) of categorization, communication, and cooperation to induce group identity. These manipulations may be considered stronger versions of Tajfel's famous "minimal group" experiments of the 1970s (Tajfel et al., 1971).

This phase of the experiment lasted approximately fifteen minutes, and the tasks were so simple that every individual and every group performed them successfully. At this point it was announced that the second stage was about to begin. In the Ingroup and In-Complex conditions the gatherings of the Blue and Red groups were dismantled and each subject was invited to seat at a computer terminal associated with her own number. In the Baseline and Complex conditions the subjects remained seated at their desks. The experimenter announced that all subjects/groups had completed successfully the first task and read a second set of instructions illustrating the mini-dictator games.

We decided not to use the strategy method for the allocation of roles in the mini-dictator 
games. ${ }^{6}$ The subjects who had picked an odd number were immediately assigned the role of dictators, and the subjects with an even number were assigned the role of recipients. After reading the instructions, the experimenter elicited questions from the audience. Once all doubts had been dispelled, the second stage of the experiment began. The dictators played the games via their computer terminals, while the recipients waited quietly at their desks. In the Baseline and Complex conditions, subjects were not associated with any group or colour. In the Ingroup and In-Complex conditions, dictators were revealed the group identity of their partners at the beginning of the game, when they saw two coloured icons (Red or Blue) associated with their own payoff and the payoff of the recipient. Since we ran only ingroup treatments, dictators were randomly matched with recipients of the same colour. Subjects received no information about other dictator/recipient matchings.

After the dictators had completed their forty-four decisions, they answered a short questionnaire concerning demographics, gender, programme of studies, plus a non-incentivised question about the motivations underlying their choices. The experimenter then asked one subject to randomly draw a number corresponding to a round of the experiment. The options for that round were announced publicly so that everybody knew what they would have earned if the dictator had chosen differently. The dictators were called to the experimenter's desk and paid privately the sum they had allocated to themselves in that round, plus the sum they had earned in the first stage of the experiment. They were then matched randomly with a recipient, and saw the experimenter put the recipient's payoff in an envelope assigned to the drawn number, together with the sum earned in the first stage. After all the dictators had been paid, the envelopes were distributed anonymously to the recipients,

\footnotetext{
${ }^{6}$ The strategy method allows the collection of a large amount of data, because every subject is asked to make a decision as if she was playing as dictator. The subjects are told who is dictator and who is recipient only later, and the money is allocated accordingly. This procedure (also called "role uncertainty") is cost-effective but has the side effect of inducing the subjects to fantasise about what they would like to happen if they were playing in a different role than the one they will effectively be assigned. In our case, dictators are likely to speculate about "what would happen if" or "how I would feel if I was to be assigned the role of recipient later in the experiment". Telling a subject right from the start that she is going to be the decision-maker, in contrast, is less likely to induce empathic feelings in dictators that may interact with the group identity effect. Iriberri and Rey-Biel (2011) find that the strategy method reduces selfish choice and increases dictators' concern for social welfare compared to a control condition with role certainty. The strategy method lowers significantly (by half) the transfer of resources in trust games (Casari and Cason, 2009). Notice that in many ways role uncertainty is similar to the "veil of ignorance" device that philosophers use to identify fair allocations or principles of distributive justice (e.g. Harsanyi, 1976; Rawls, 1971).
} 
and all the subjects were invited to leave the room. The whole procedure was described in detail in the instructions and was common knowledge among subjects before they were asked to make their decisions.

The experiment was implemented with z-Tree (Fischbacher, 2007) and lasted approximately 70 minutes. We ran nine sessions in three separate occasions (January 2012, February 2013, and October 2014). The 256 subjects were inexperienced volunteers from the student population of the University of Parma, predominantly undergraduates in the Department of Economics. Among the dictators, male subjects constituted 56.45 per cent of the sample. The average earning for the whole experiment (first and second stage) was 13.37 euro (14.8 euro for the dictators, 11.94 euro for the recipients). From now on we shall ignore the money earned in the first stage (memory task) and concentrate on the allocations chosen in the mini-dictator games.

\section{Results}

In this section we present the findings of our experiment. Section 4.1 compares our results with those obtained in the literature using social preference models. Both our experiment and Chen and Li (2009) show that the effect of group identity on social preferences is rather weak. In Section 4.2 we disaggregate our data and analyse the impact of group identity across types of games and treatments. We use several different statistical tools, and we conclude that the evidence supports the interpretation of group identity in terms of heuristic.

\subsection{Group identity as social preference: a reappraisal}

In the study that is closest to our experiment, Chen and Li (2009) measure the effect of group identity using a simple social preference model devised by Charness and Rabin (2002). In this sub-section we spend some time analysing Chen and Li's seminal results, to illustrate the difficulties of estimating the net effect of group identity on social preferences. This, in turn, will help us show in which way our study constitutes an improvement with respect to previous experiments, and how it helps understand the group identity effect better.

Charness and Rabin (2002) estimate the following utility function for the dictator 


$$
u_{d}\left(\pi_{r} ; \pi_{d}\right)=w \pi_{r}+(1-w) \pi_{d}
$$

where the parameter $w$ measures the weight of pro-social motives, i.e. the concern of dictators for the payoff of the recipient $\left(\pi_{r}\right)$. Notice that this utility function is based on the strong assumption that $\pi_{d}$ and $\pi_{r}$ are perfect substitutes. Charness and Rabin (2002) and Chen and Li (2009) allow the parameter $w$ to vary depending on whether the payoff of the dictator is higher or lower than the payoff of the recipient. In practice, the utility function is specified as follows:

$$
u_{d}\left(\pi_{r} ; \pi_{d}\right)=(\rho r+\sigma s) \pi_{r}+[1-(\rho r+\sigma s)] \pi_{d}
$$

where $\rho$ is a "charity" coefficient that applies when the payoff of the dictator is higher than that of the recipient $(r=1, s=0)$, while $\sigma$ is an "envy" coefficient that applies when the payoff of the dictator is lower than that of the recipient $(r=0, s=1) .{ }^{7}$ Although this specification allows the choices to be game-specific to some extent, it also implies that preferences are stable across different types of games. As we shall see, there are good reasons to doubt that this assumption holds. For example the games in Table 2 are treated as observationally equivalent by this estimation procedure, in spite of the fact that different motivations are likely to be at work in each case $\left(\pi_{r}=\pi_{d}\right.$ in Option A, while $\pi_{r}>\pi_{d}$ in Option $B$, so $r_{A}=0, s_{A}=0, r_{B}=1, s_{B}=0$ in all five cases).

\begin{tabular}{cc|cc|c}
\hline \multicolumn{3}{c}{ Option A } & \multicolumn{3}{c}{ Option B } \\
$\pi_{d}$ & $\pi_{r}$ & $\pi_{d}$ & $\pi_{r}$ & Our classification \\
\hline 1000 & 1000 & 1400 & 800 & Expensive Giving \\
1000 & 1000 & 1200 & 800 & Zero-Sum Giving \\
1000 & 1000 & 1200 & 600 & Cheap Giving \\
1000 & 1000 & 800 & 600 & Spite \\
1000 & 1000 & 1000 & 800 & Envy \\
\hline
\end{tabular}

Table 2: Five mini-dictator games

\footnotetext{
${ }^{7}$ Note that our definition of envy differs from that in Charness and Rabin (2002), as we classify as envy games those in which the dictator can increase the payoff of the recipient at no cost, but without any restriction on the relative levels of the payoffs.
} 
Chen and Li (2009) estimate the following values for the parameters of the CharnessRabin model:

$$
\begin{gathered}
u_{d}\left(\pi_{r} ; \pi_{d}\right)=0.427 \pi_{r}+0.573 \pi_{d}, \text { when } \pi_{r}>\pi_{d} \\
u_{d}\left(\pi_{r} ; \pi_{d}\right)=-0.049 \pi_{r}+1.049 \pi_{d}, \text { when } \pi_{r}<\pi_{d}
\end{gathered}
$$

Group identity is modelled by means of two dummies, $a$ and $b$, that capture the additional impact of the Ingroup manipulation on the charity and envy parameters, respectively:

$$
u_{d}\left(\pi_{r} ; \pi_{d}\right)=[(1+a) \rho r+(1+b) \sigma s] \pi_{r}+\{1-[(1+a) \rho r+(1+b) \sigma s]\} \pi_{d}
$$

Chen and Li (2009) estimate this equation with decisions taken in the Ingroup and Outgroup conditions, finding that the weight assigned to the recipient's payoff in the Ingroup condition is significantly higher than in the Outgroup condition. (The charity parameters are 0.474 and 0.323 , while the envy parameters are -0.08 and -0.112 , respectively.)

Making comparisons across Ingroup and Outgroup conditions magnifies the effect of group identity, for one is effectively combining an Outgroup with an Ingroup effect. For this reason it is preferable to measure the net effect of the Ingroup manipulation, taking a control condition without groups as our reference point. If we use the data in Chen and $\mathrm{Li}$ (2009) to estimate the parameters of the Ingroup choices against the Baseline condition, unsurprisingly, there is a less pronounced difference across treatments (see Table 3). Notice that in this case only the identity parameter $a$ is significantly different from zero (at $10 \%$ ) determining a charity parameter in the Ingroup condition (0.482) that is significantly higher than in the Baseline (0.428). In contrast, the identity parameter $b$ turns out to be not significantly different from zero, implying that the envy parameters are not distinguishable from each other in the two treatments.

A second concern is that Chen and Li's estimates are based on a mixed set of data that does not contain only the results of mini-dictator games. Among twenty-four games, their data-set includes nineteen sequential "response" game where one player (analogous to the "recipient") has the opportunity to make a preliminary move that affects the final allocation of payoffs. In these games clearly the second mover cannot be equated to that of a pure 


\begin{tabular}{|c|c|c|c|c|c|c|}
\hline & \multicolumn{2}{|c|}{ Baseline } & \multicolumn{2}{|c|}{ Ingroup } & \multicolumn{2}{|c|}{ Identity parameters } \\
\hline & Charity: $(\rho)$ & Envy: $(s)$ & Ch: $\rho(1+a)$ & En: $s(1+b)$ & $a$ & $b$ \\
\hline $\begin{array}{l}\text { Chen and } \mathrm{Li}(2009) \\
\text { all data }(\mathrm{N}=999)\end{array}$ & $0.428^{* *}$ & $-0.052^{* *}$ & $0.482^{* *}$ & $-0.024^{*}$ & $0.126^{*}$ & -0.534 \\
\hline $\begin{array}{l}\text { Chen and } \mathrm{Li}(2009) \\
\text { minidict. }(\mathrm{N}=196)\end{array}$ & $0.285^{*}$ & -0.219 & $0.415^{* *}$ & $0.172^{*}$ & 0.456 & -1.789 \\
\hline $\begin{array}{l}\text { Our data } \\
(\mathrm{N}=2816)\end{array}$ & $0.230^{* *}$ & $-0.074^{* *}$ & $0.254^{* *}$ & -0.086 & 0.105 & 0.167 \\
\hline
\end{tabular}

Table 3: Estimates of the Charness-Rabin model with different samples

dictator, because decisions are likely to be influenced by reciprocity concerns. Therefore, in order to get an estimate of subjects' attitudes toward "pure" distributive choice, we have estimated the social preference model again on Chen and Li's data, but using only their five bona fide mini-dictator games. The absence of reciprocity reduces the weight of the recipient's payoff in the Baseline treatment (see the second row of Table 3). The number of observations however is reduced drastically (about $20 \%$ of the original sample), which certainly contributes to make the identity parameters statistically indistinguishable.

The last row of Table 3 replicates the same exercise using the full set of forty-four minidictator games in our Ingroup and Baseline conditions. Notice that the charity parameter in our Baseline condition is lower than Chen and Li's (all data) while it is of the same order of magnitude as the parameter estimated using their subsample of minidictators. The envy parameter is instead closer to the full set of games in Chen and Li (2009). The weights of the recipient's payoff look stable across our experimental treatments. In fact, the identity parameters are rather low and not significantly different from zero. This result suggests either that the impact of group identity on distributive preferences is modest, or that the effect of group identity is not captured by this estimation procedure.

The point of this analysis is not to question the general picture emerging from Chen and Li's measures of the impact of group identity on social preferences, but to illustrate how difficult it is to obtain a meaningful estimate by mixing the data of a small number of experimental games. Overall, we suspect that aggregate data provide limited information regarding the motives that drive dictators' behaviour. To achieve better insights it is necessary to look separately at classes of games that offer similar trade-offs among egalitarian, 
altruistic, selfish, and efficient options. In the next section we proceed gradually, moving from relatively broad to more refined classifications.

\subsection{Group identity as heuristic}

In this sub-section we analyze data across treatments and types of games. The results lend support to the interpretation of group identity as the trigger of a particular heuristic rather than as a manipulation of social preferences. Section 4.2.1 presents simple summary statistics and non-parametric tests. In Section 4.2.2 and 4.2.3 we use multivariate analysis to look at the main determinants of behaviour across treatments and types of game, respectively.

\subsubsection{Summary statistics}

Table 4 reports choice frequencies and non-parametric tests across treatments for five different types of mini-dictator games. We have 32 independent observations and roughly 1400 decisions for each treatment. In the Complex conditions the overwhelming majority of decisions (95\%) converged on one of the two options shared in common with the other treatments, so we eliminate the dominated choices from our analysis. ${ }^{8}$

Starting from the top row, the games are listed in order of decreasing cost of altruism. In the class of so-called "giving" games the payoff of the recipient can only be increased at a cost for the dictator. In Expensive, Zero-Sum, and Cheap Giving games, each donated unit costs respectively two units, one unit, and half a unit to the dictator. In Envy games the payoff of the dictator is invariant across the options (giving is costless and not giving thus may be motivated by envy). In Spite games, finally, altruism is advantageous and not giving is costly for the dictator. Across all conditions the data suggest that choices are influenced not only by the cost of giving, but also by the relative rank of dictator and recipient's payoffs: when the dictator's payoff is larger regardless of her choice, we shall say that the game is "favourable" to the dictator. When giving implies a larger payoff for the recipient, we shall say that altruism is "unfavourable" to the dictator.

\footnotetext{
${ }^{8}$ Four subjects were responsible for more than half $(58.6 \%)$ of the dominated choices, suggesting a high degree of confusion. Without these four subjects, dominated options account for only $2.2 \%$ of the choices. Replicating the analysis without these "confused" subjects does not affect the results in any significant way.
} 
Significance is calculated with a battery of Fisher exact tests. The tests evaluate whether the fraction of subjects making an altruistic choice in each type of game differs significantly from what is observed in another condition. Table 4 contains four pairwise comparison: Baseline Vs. Ingroup, Complex Vs. In-Complex, Baseline Vs. Complex, and Ingroup Vs. InComplex. For instance, the fraction of subjects making an altruistic choice in the Expensive Giving Favourable games is significantly higher in the Ingroup compared both to the Baseline and to the In-Complex conditions, while this is not the case comparing the Complex with the In-Complex and the Baseline treatments.

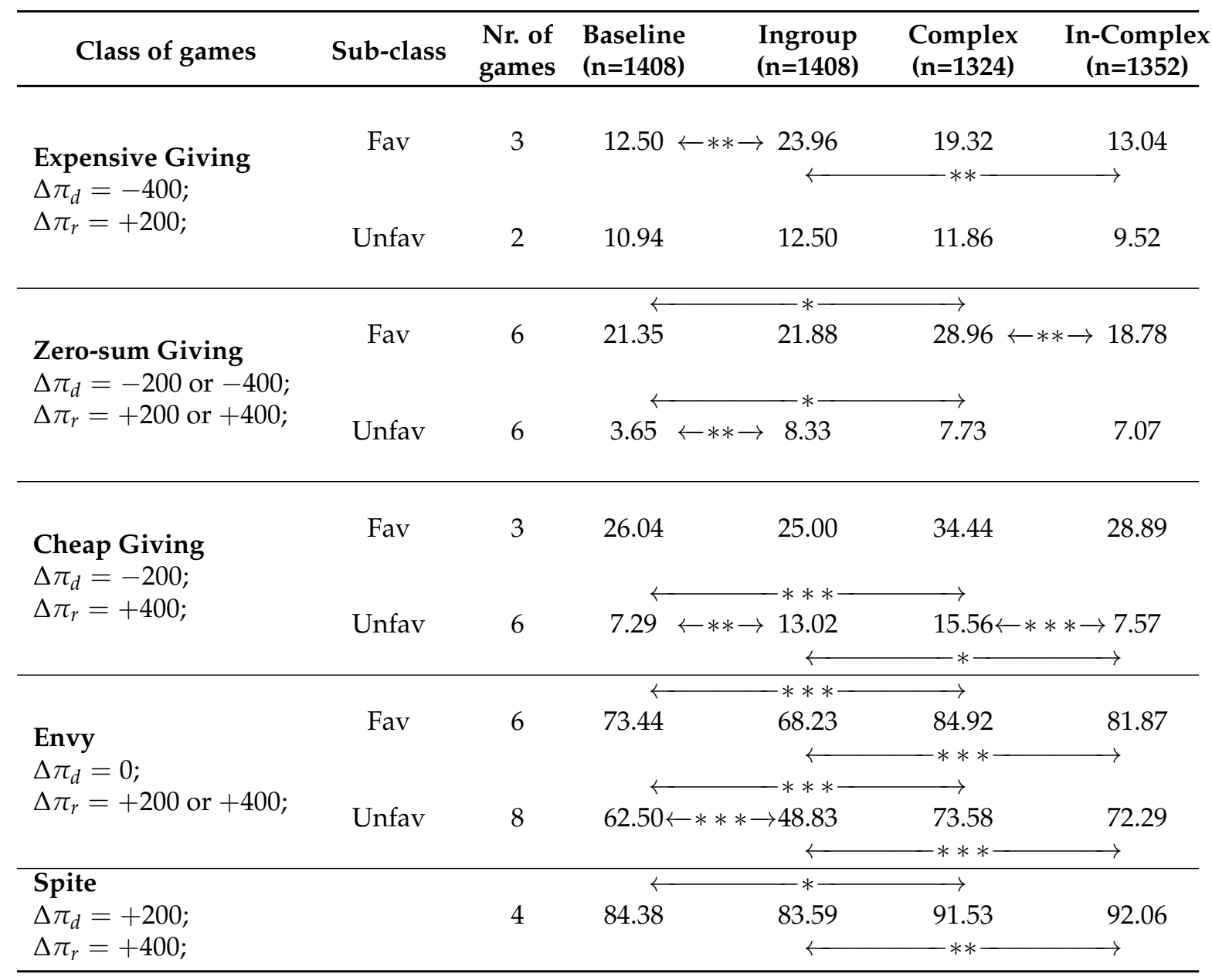

The figures represent the frequency with which the most favourable option for the recipient is chosen. $\Delta \pi_{d}$ and $\Delta \pi_{r}$ represent the change in the payoffs of dictator and recipient. Significance thresholds obtained with a battery of Fisher tests: ${ }^{*}=0.1,{ }^{* *}=0.05,{ }^{* * *}=0.01$.

Table 4: Summary of results per class of games, and according to treatment. 
We begin with Giving games, which have already been the subject of analysis in previous experiments, most of which report an increase of donations in the Ingroup compared to the Baseline condition. Such a result holds in our giving games, although the difference reaches statistical significance only in some sub-classes of games. Note further that if altruism results in an unfavourable allocation, dictators are much less likely to give to the recipient. As soon as we extend the analysis to Envy and Spite games, however, the effect of group identity changes drastically. In Envy games the group identity manipulation increases the probability that the dictator withdraws money from the recipient, even though choosing the altruistic option would not reduce the payoff of the dictator. Moreover, the effect holds both when the difference in payoffs is favourable to the dictator, and when it is unfavourable. In so-called Spite games, choosing the altruistic option is advantageous to the dictator, so unsurprisingly this allocation is chosen by a large majority of subjects. The effect of group identity is insignificant.

These data confirm that it is important to use a wide range of mini-dictator games. Although it is possible to estimate consistent and robust social preference functions by restricting the analysis to a homogeneous class of games, the parameter specifications are fragile when we move across different classes.

The second result that emerges from Table 4 is the effect of complexity. Recall that subjects in this condition are offered a choice between four options, two of which are dominated and hence in principle irrelevant for a rational decision maker. The analysis of reaction times unsurprisingly indicates that subjects spend more time to make each decision in the Complex and In-Complex condition than in the Baseline and In-group conditions. ${ }^{9}$ However, complexity also increases generosity in most of the classes, as shown by the comparison between the Complex and the Baseline conditions, particularly when it is associated with a gain in terms of efficiency. Although we lack data that could support this hypothesis directly, the psychological literature on the attraction effect (Simonson, 1989) suggests that subjects may follow a heuristic based on the systematic search of Pareto-improvements (or

\footnotetext{
${ }^{9}$ Reaction times increase roughly from seven to eleven seconds in the two complex treatments. Interestingly, subjects take significantly more time to make altruistic choices, compared to selfish ones. Group identity reduces the time of altruistic choice only in Giving games (where it increases generosity), which suggests that it does indeed function as a "fast and frugal" heuristic. Data about reaction times are available upon request.
} 
the elimination of dominated options). The elimination of dominated options, once triggered, may induce dictators to discard systematically all inefficient allocations, and hence the attraction effect seems to be strengthened in the complex conditions.

A similar effect is observed in Spite games. In these games dictators have the opportunity to lower the payoff of the recipient at a cost for themselves. The frequency of spiteful choices is low but still noteworthy (around 16\%) in the Baseline and in the Ingroup conditions. Complexity however leads to a significant reduction of spiteful behaviour. Again, this is unsurprising if the dictators follow the strategy of eliminating dominated options: spite is always inefficient and it is systematically avoided by dictators who adopt this decision rule.

Crucially, our data show that complexity destroys the effect of group identity that has been observed in the Ingroup treatment. The mere presence of a pair of dominated allocations is sufficient to reverse the effect of group membership. Both the increased pro-sociality in the Giving games and the envy-inducing effect of group identity are swept away. Choices in In-Complex become indistinguishable from those in the Complex treatment across the board, with two puzzling exceptions within Giving games in which the fraction of altruistic choices is significantly lower than in the Complex and more in line with the Baseline condition. This finding suggests that subjects follow a completely different decision heuristic.

\subsubsection{Multivariate analysis}

To back up these insights, we run some regressions on the entire sample of data $(\mathrm{N}=5492$, i.e. 44 choices of 128 dictators, disregarding 140 dominated choices). The multivariate specification that can be implemented is limited for two reasons. The first is the linear dependence of determinants. For instance, it is impossible to disentangle at the same time the role played by variations in the dictator's payoff (self-interest) from the role of variations in the recipient's payoff (altruism) and from the level of efficiency, because one measure is by construction a linear combination of the other two. As a result, we are forced to choose a parsimonious specification in which the degrees of freedom for each choices are limited to:

1. Variations in dictator's payoff (Self), defined as the difference between the dictator's payoff in Option A minus his payoff in Option B;

2. Variations in recipient's payoff $(A l t)$, defined as the difference between the recipient's payoff in Option A minus his payoff in Option B; 
3. Inequality in one of the two options (Diff), defined as the difference between the dictator's and the recipient's payoff in (the arbitrarily chosen) Option A.

Subjects' choices may of course differ according to how these factors are combined (i.e. the type of game), according to the characteristics of the treatment, and as a function of individual characteristics. In other words, the payoff features (1-3) can in principle be interacted with other variables to enrich the empirical specification.

Apart from obvious dimensionality problems induced by the introduction of a complete set of interactions, such an exercise is also limited by the experimental design. Our list of forty-four choices covers five types of game, different signs and degrees of inequality, as well as different levels of payoffs. Although wide, the set of choices does not ensure enough variance to disentangle all the possible interactions between the determinants. For instance, it is not possible to interact the effect of Self with that of the type of game, because the two are highly correlated. Only within the Zero-Sum Giving games there is some variance in the change of dictator's payoffs; the difference in dictator's payoffs is always 400 units in Expensive Giving games, 200 units in Cheap Giving and in Spite games, and zero units in Envy games. Hence, we cannot disentangle whether the likelihood of making a selfish choice depends on the type of game rather than on the size of Self, because a higher variance in the change of dictator's payoffs within each type of game would be required to accomplish this task. $^{10}$

To ensure a meaningful interpretation of the coefficients we use as dependent variable the probability of choosing the left option in each choice (see Table 1 for a summary of choices). ${ }^{11}$

To exploit the longitudinal nature of the data we estimate a fixed effect model controlling for any observable and unobservable individual characteristic. We fully exploit the degrees of freedom that are available, including among the explanatory variables the variation in

\footnotetext{
${ }^{10}$ Besides limiting our empirical specification, notice that this feature also imposes a constraint on the number of parameters that can be meaningfully included in the model. This problem is present a fortiori in earlier experiments that try to estimate social preference functions using fewer games with less variance in payoffs than ours.

${ }^{11} \mathrm{Had}$ we chosen instead the probability of making the choice that maximizes the dictator's payoff, most of the effect of the Self coefficient would have been captured by the constant. A similar argument would apply to the Alt coefficient in case we had chosen as dependent variable the probability of making the choice that maximizes the recipient's payoff.
} 
the payoff of the dictator (Self) and of the recipient (Alt), as well as the difference between self/other payoffs in the left option (Diff). To increase psychological realism, we allow for a different reaction between positive and negative differences in the level of payoffs (Posdiff when $\pi_{d}>\pi_{r}$ Vs. Negdiff when $\pi_{d}<\pi_{r}$ ), as well as for perfectly equal payoffs (NodiffA, NodiffB). ${ }^{12}$ We then estimate a linear probability model, so that coefficients can straightforwardly be read as percentage changes $(0.01=1 \%)$.

Column 1 of Table 5 shows what happens when these variables are regressed on our entire data-set. Notice that the probability of choosing a given option increases with the change in payoffs of both players, although unsurprisingly the effect is stronger for one's own payoffs. The coefficient of 0.00149 for Self means that the likelihood of choosing Option A increases by $14.9 \%$ for every euro of difference in $\pi_{r}$ between Option A and Option B. Second, dictators react negatively as the difference in the payoffs increases, regardless of its sign. The dummy variable NodiffB substantially increases the likelihood that Option B is preferred.

As a second step, we allow some of these explanatory variables to have a different impact across treatments, by means of interaction terms with our Baseline, Ingroup, Complex, and In-Complex conditions. Since the interaction terms with the difference in the level of the payoffs (Posdiff and Negdiff) turn out to be systematically non-significant, we have removed them from the estimated models.

The regressions reveal that the effect of self-interest is different across treatments. It is significantly lower in the Ingroup conditions, with group identity that seems to reduce selfish concerns, consistently with common wisdom. However, adding the dominated options more than counterbalances this effect: in fact, the sum of the coefficients of the interaction terms (In, Complex, and In-Complex) is significantly positive (F-test, $p=0.052$ ). The effect of altruism is also idiosyncratic, although unsurprisingly its size is much smaller across the board. What is truly surprising is that group identity alone seems to reduce altruistic concerns. Again, the sum of the coefficients of the interaction terms (In, Complex, and InComplex) is significantly positive (F-test, $p<0.001$ ). The impact of the difference in the

\footnotetext{
${ }^{12}$ Notice that these coefficients cannot be interpreted in terms of inequality aversion because we do not take into account the difference in the payoffs of the alternative option.
} 
level of payoffs barely changes, compared to the first regression. ${ }^{13}$ Overall, these results confirm the findings reported in the previous section, i.e. that the effects of group identity are not robust, since a small perturbation of the task is sufficient to make them disappear. However, in order to better understand how complexity affects group identity it is necessary to distinguish between different types of games.

\begin{tabular}{|c|c|c|}
\hline & \multicolumn{2}{|c|}{$\begin{array}{l}\text { Dependent Variable: Choice of left option } \\
(1)\end{array}$} \\
\hline Self & $\begin{array}{l}0.00149 * * * \\
{[51.21]}\end{array}$ & $\begin{array}{l}0.00150 * * * \\
{[27.54]}\end{array}$ \\
\hline Self*In & & $\begin{array}{l}-0.00022 * * * \\
{[2.96]}\end{array}$ \\
\hline Self*Complex & & $\begin{array}{c}0.00005 \\
{[0.60]}\end{array}$ \\
\hline Self*In*Complex & & $\begin{array}{l}0.00033^{* * *} \\
{[3.01]}\end{array}$ \\
\hline Alt & $\begin{array}{c}0.00025^{* * *} \\
{[12.10]}\end{array}$ & $\begin{array}{l}0.00018^{* * *} \\
{[4.47]}\end{array}$ \\
\hline Alt $^{*}$ In & & $\begin{array}{l}-0.00012 \text { ** } \\
{[2.26]}\end{array}$ \\
\hline Alt*Complex & & $\begin{array}{l}0.00026^{* * *} \\
{[4.72]}\end{array}$ \\
\hline Alt ${ }^{*} \operatorname{In}^{*}$ Complex & & $\begin{array}{c}0.00005 \\
{[0.63]}\end{array}$ \\
\hline Posdiff & $\begin{array}{l}-0.00031^{* * *} \\
{[12.57]}\end{array}$ & $\begin{array}{l}-0.00031^{* * *} \\
{[12.52]}\end{array}$ \\
\hline Negdiff & $\begin{array}{l}-0.00033^{* * *} \\
{[9.10]}\end{array}$ & $\begin{array}{l}-0.00033^{* * *} \\
{[9.10]}\end{array}$ \\
\hline NodiffA & $\begin{array}{c}0.00415 \\
{[0.17]}\end{array}$ & $\begin{array}{c}0.03837 \\
{[0.16]}\end{array}$ \\
\hline NodiffB & $\begin{array}{l}-.08836^{* * *} \\
{[6.14]}\end{array}$ & $\begin{array}{l}-0.08831^{* * *} \\
{[6.18]}\end{array}$ \\
\hline$\overline{\mathrm{N}}$ & 5492 & 5492 \\
\hline Adj. $R^{2}$ & 0.351 & 0.360 \\
\hline
\end{tabular}

\footnotetext{
${ }^{13}$ Results are robust to Probit and Logit specifications, with the only small exception of the coefficient of NodiffA that becomes significant in the Logit model.
} 


\subsubsection{Multivariate analysis by type of game}

In this section we appraise the robustness of the group identity effect across different types of games. The constraints imposed by design become even more binding at this point, because the variance of the regressors across different classes of games cannot be exploited any longer. As a consequence, the estimations that follow do not include the same variables as in Table 5. For instance, in Giving games there is a strong negative correlation between the payoffs of the two players. Hence, it would not be meaningful to include both Self and Alt variables in the regression. For this reason we include in the regression of Table 6 only the opportunity cost of giving that is implicit in each choice (Oppcost=Self/Alt). Notice that the opportunity cost of the altruistic choice distinguishes Cheap from Zero-sum and from Expensive games, as the absolute value of Oppcost is lower than, equal to, and greater than one, respectively.

The effect of the opportunity cost of making an altruistic choice in Giving games is in line with ex ante expectations, as shown in the first column of Table 6. A 100\% increase of the price of giving implies a 33\% decrease in the probability of giving. Group identity makes the altruistic choice significantly more likely, ceteris paribus, and so does adding the dominated choices to the Baseline. In contrast, these effects vanish completely when complexity and group identity interact. The sum of the coefficients of the interaction terms (In, Complex, and In-Complex) is not significantly different from zero (F-test, $p=0.802)$. Differences in the level of dictator's and recipient's payoffs still play a significant role (unfavorable differences even more so), and the absence of inequality turns out to be particularly relevant.

The second column of Table 6 provides a useful comparison with those games in which the payoff of the dictator does not change across the available options, i.e. so-called Envy games. In this case the variable self has obviously been omitted as it is constant and equal to zero, and therefore the probability of making an altruistic choice only depends on the payoff of the recipient and on the difference between the payoffs. Altruism exerts a significant effect, while the role played by differences in the payoffs is less clear-cut. The correlation in the Ingroup condition is significantly negative. This means that in the Ingroup condition subjects are less likely to benefit their counterpart for free than in the Baseline, i.e. the opposite effect than the one we would have expected. Adding the two dominated options has 


\begin{tabular}{|c|c|c|}
\hline & \multicolumn{2}{|c|}{ Dependent Variable: Choice of left option } \\
\hline & Giving games & Envy games \\
\hline Oppcost & $\begin{array}{c}-0.33581^{* * *} \\
{[26.98]}\end{array}$ & \\
\hline Oppcost*In & $\begin{array}{c}0.03504^{* *} \\
{[2.15]}\end{array}$ & \\
\hline Oppcost ${ }^{*}$ Complex & $\begin{array}{c}0.04373^{* * *} \\
{[2.64]}\end{array}$ & \\
\hline Oppcost ${ }^{*} \operatorname{In}^{*}$ Complex & $\begin{array}{c}-0.07464^{* * *} \\
{[3.19]}\end{array}$ & \\
\hline Alt & & $\begin{array}{c}0.00046^{* * *} \\
{[6.51]}\end{array}$ \\
\hline Alt ${ }^{*}$ In & & $\begin{array}{c}-0.000311^{* * *} \\
{[3.22]}\end{array}$ \\
\hline Alt*Complex & & $\begin{array}{c}0.00036^{* * *} \\
{[3.67]}\end{array}$ \\
\hline Alt*In*Complex & & $\begin{array}{c}0.00025 * \\
{[1.82]}\end{array}$ \\
\hline Posdiff & $\begin{array}{c}-0.00027^{* * *} \\
{[9.77]}\end{array}$ & $\begin{array}{c}-0.00013 \text { * } \\
{[1.65]}\end{array}$ \\
\hline Negdiff & $\begin{array}{c}-0.00048^{* * *} \\
{[12.28]}\end{array}$ & $\begin{array}{c}-0.00010 \\
{[0.91]}\end{array}$ \\
\hline NodiffA & $\begin{array}{c}0.15219^{* * *} \\
{[3.97]}\end{array}$ & \\
\hline NodiffB & $\begin{array}{c}-0.07807^{* * *} \\
{[4.11]}\end{array}$ & $\begin{array}{c}-0.09316^{* * *} \\
{[3.63]}\end{array}$ \\
\hline $\mathrm{N}$ & 3240 & 1752 \\
\hline Adj. $R^{2}$ & 0.430 & 0.168 \\
\hline
\end{tabular}

Absolute t-statistics in brackets. The variable nodiffleft is not included in Column 2 because (by chance) there is no envy game without difference in the payoffs in the left option. Significance thresholds : ${ }^{*}=0.1,{ }^{* *}=0.05$, $* * *=0.01$

Table 6: Fixed-effects estimates (by type of game)

both the effect of increasing efficiency, and that of reversing the envious behaviour observed in the Ingroup treatment.

As far as Spite games are concerned, it is not possible to estimate a model delivering more insights than the one in Table 4, due both to the linear combination between the change of payoffs of dictators and recipients, and to the relatively low number of games of this type. Once we control for (Posdiff and Negdiff the only coefficient approaching traditional significance levels is that of Complex confirming the use of an efficiency-enhancing decision rule. In this case no role is played by group identity.

The findings seem to indicate that the effect of group identity cannot be accounted for 
in terms of a (regular) shift in social preference parameters. First, the Ingroup manipulation induces a change in behaviour that varies considerably across different types of games. Second, this effect is not robust to an increase of complexity caused by the addition of two dominated options.

\section{Conclusion}

The experiment was designed to achieve two goals: first, we aimed to extend the range of mini-dictator games used in the literature, so as to map the effect of group identity in a broader space of self-other allocations. Second and foremost, we intended to test the robustness of social preference models and to explore an alternative account of group identity as a framing effect (Bacharach, 2006; Tajfel, 1982).

On the first front, we have seen that the effect of group identity on distributive choice is modest overall and highly variable across types of games. Charness and Rabin's model of social preference, used by Chen and Li (2009), is unable to capture the impact of group identity on the whole set of games, a null result that we explain as a composition effect. This failure confirms our ex ante conjecture that it is important to extend the experimental design to cover choices that do not imply a strictly positive trade-off between dictator's and recipient's payoff. Moreover, the results cast a shadow of doubt on the robustness of the effect of group identity across different games.

A further insight is derived by introducing a minor change in the environment that, in principle, should not affect the behaviour of dictators. In the complex conditions subjects are exposed to the same group identity manipulation, exactly as in the Ingroup vs. Baseline treatments, but dictators are asked to choose among a larger set of options that includes two additional dominated allocations. We observe that this manipulation of complexity increases efficiency across the board, compared to the simple Baseline condition. This result shows that social preferences in general are not perfectly stable, something that is already known from different studies (Bardsley, 2008; List, 2007). However, when complexity is combined with identity, the change of behavior is of a different nature, since we observe a complete reversal of the ingroup effect. Surprisingly, in a complex environment group identity either has no impact, or has the opposite effect than what we observe in the simple 
task, thereby violating minimal regularity requirements.

These data suggest that group identity, when it is induced in the laboratory, is fragile to minor variations in the choice menu. Since preferences and utility functions, as they are usually understood in economics, are supposed to capture regular patterns, our data indicate that the effect of group identity should not be included in agents' utility functions (contrary to what is suggested by Akerlof and Kranton (2010) and others). In our view the data also lend plausibility to a specific hypothesis that deserves more attention than it has received so far: group identity manipulations do not change individual preferences, replacing selfish motives with a concern for the common good. Rather, they trigger a set of heuristic rules that induce different behaviours (generous and/or inequality reducing) across different types of games.

Like other heuristics that have been studied in the psychological and economic literature, group identity provides guidance in an unfamiliar experimental situation and allows subjects to economize cognitive effort. However, it competes for cognitive salience with alternative rules of thumb and hence may be easily displaced by small changes in the decision environment. Our complex conditions were designed to explore this scenario. The addition of dominated options either erased the effect of group identity or induced diametrically opposite behaviour. It is possible - perhaps likely - that the mere presence of dominated options made an alternative decision heuristic salient, as shown by psychological studies of the so-called "attraction effect" (Huber and Puto (1982), Simonson (1989)). When this heuristic (elimination of dominated choices) was adopted by the dictators, the effect of group identity disappeared or changed direction altogether.

\section{References}

Akerlof, G., Kranton, R., 2000. Economics and identity. Quarterly Journal of Economics 115, 715-753.

Akerlof, G., Kranton, R., 2010. Identity Economics: How Our Identities Shape Our Work, Wages, and Well-Being. Princeton University Press.

Andreoni, J., Miller, J., 2002. Giving according to garp: An experimental test of the consistency of preferences for altruism. Econometrica 70, 737-753.

Bacharach, M., 2006. Beyond Individual Choice: Teams and Frames in Game Theory. Princeton University Press. 
Bardsley, N., 2008. Dictator game giving: altruism or artefact? Experimental Economics 11, 122-133.

Benjamin, D., Choi, J., Strickland, A., 2010. Social identity and preferences. American Economic Review 100, 1913-1928.

Bernhard, H., Fehr, E., Fischbacher, U., 2006. Group affiliation and altruistic norm enforcement. American Economic Review 96, 217-221.

Bhatia, S., 2013. Distributional preferences in groups, unpublished paper, Carnegie Mellon University.

Brewer, M., Kramer, R., 1986. Choice behavior in social dilemmas: Effects of social identity, group size, and decision framing. Journal of Personality and Social Psychology 50, 543-549.

Camerer, C., 2003. Behavioral Game Theory. Princeton University Press.

Casari, M., Cason, T., 2009. The strategy method lowers measured trustworthy behaviour. Economics Letters $103,157-159$.

Charness, G., Rabin, M., 2002. Understanding social preferences with simple tests. Quarterly Journal of Economics $117,817-869$.

Charness, G., Rigotti, L., Rustichini, A., 2007. Individual behavior and group membership. American Economic Review 97, 1340-1362.

Chen, Y., Li, S., 2009. Group identity and social preferences. The American Economic Review 99 (1), $431-457$.

Cooper, D., Kagel, J., 2005. Are two heads better than one? team versus individual play in signaling games. American Economic Review 95, 477-509.

Eckel, C., Grossman, P., 2005. Managing diversity by creating team identity. Journal of Economic Behavior and Organization 58, 371-392.

Efferson, C., Lalive, R., Fehr, E., 2008. The coevolution of cultural groups and ingroup favoritism. Science 26, 1844-1849.

Fehr, E., Schmidt, K., 2006. The economics of fairness, reciprocity and altruism-experimental evidence and new theories. In: Kolm, S., Ythier, J. (Eds.), Handbook of the Economics of Giving, Reciprocity and Altruism. Elsevier.

Filippin, A., Guala, F., 2013. Costless discrimination and unequal achievements in an experimental tournament. Experimental Economics 16, 285-305.

Fischbacher, U., 2007. z-tree: Zurich toolbox for ready-made economic experiments. Experimental Economics 10, 171-178. 
Forsythe, R., Horowitz, J.L., S. N., Sefton, M., 1994. Fairness in simple bargaining experiments. Games and Economic Behavior 6, 347-369.

Gigerenzer, G., Todd, P., the ABC Research Group, 2000. Simple Heuristics that Make Us Smart. Oxford University Press.

Goette, L., Huffman, D., Meier, S., Sutter, M., 2010. Group membership, competition and altruistic versus antisocial punishment: evidence from randomly assigned army groups. IZA Discussion Paper No. 5189.

Guala, F., Mittone, L., Ploner, M., 2013. Group membership, team preferences, and expectations. Journal of Economic Behavior and Organization 86, 183-190.

Güth, W., Ploner, M., Regner, T., 2009. Determinants of in-group bias: is group affiliation mediated by guilt aversion? Journal of Economic Psychology 30, 814-827.

Hargreaves Heap, S., Zizzo, D., 2009. The value of groups. American Economic Review 99, 295-323.

Harsanyi, J. C., 1976. Essays on Ethics, Social Behavior, and Scientific Explanation. Reidel.

Huber, J.J., P. J., Puto, C., 1982. Adding asymmetrically dominated alternatives:violations of regularity and the similarity hypothesis. Journal of Consumer Research 9, 90-98.

Iriberri, N., Rey-Biel, P., 2011. The role of role uncertainty in modified dictator games. Experimental Economics $14,160-180$.

Kahneman, D., Knetsch, J., Thaler, R., 1986. Fairness and the assumptions of economics. Journal of Business 59, S285-S300.

Krupka, E. L., Weber, R. A., 2013. Identifying Social Norms Using Coordination Games: Why Does Dictator Game Sharing Vary? Journal of the European Economic Association 11 (3), 495-524.

List, J., 2007. On the interpretation of giving in dictator games. Journal of Political Economy 115, 482-493.

Rawls, J., 1971. A Theory of Justice. Oxford University Press.

Ruffle, B., Sosis, R., 2006. Cooperation and the in-group-out-group bias: a field test on israeli kibbutz members and city residents. Journal of Economic Behavior and Organization 60, 147-163.

Sherif, M., H. O. W. B. H. W., Sherif, C., 1961. Intergroup conflict and cooperation: the robbers cave experiment. University of Oklahoma Book Exchange.

Simonson, I., 1989. Choice based on reasons: the case of attraction and compromise effect. Journal of Consumer Research 16, 158-174.

Sugden, R., 2000. Team preferences. Economics and Philosophy 16, 174-204. 
Sutter, M., 2009. Individual behavior and group membership. American Economic Review 99, 2247-2257.

Tajfel, H., 1982. Human Groups and Social Categories. Cambridge University Press.

Tajfel, H., Billig, M., Bundy, R., Flament, C., 1971. Social categorization and intergroup behaviour. European Journal of Social Psychology 1, 149-178.

Tversky, A., Kahneman, D., 1986. Rational Choice and the Framing of Decisions. Journal of Business 59(4), 251278.

Zizzo, D. J., 2011. You are not in my boat: common fate and discrimination against outgroup members. International Review of Economics 58, 91-103.

\section{Appendix: Experimental instructions}

[The main text is a literal translation of the original instructions (in Italian) for the Baseline condition; whenever the instructions differed in the Ingroup or in the complex conditions, we have inserted the relevant paragraphs between brackets.]

\subsection{Stage 1}

You are about to take part in an experiment financed for research purposes by various foundations. The experiment is divided in two phases. At the beginning of each stage you will receive instructions that will explain the task you will face. Throughout the experiment it will be prohibited to speak or help other participants. If you have any doubt or ques-

tion please ask the assistants. If you do not follow this rule, we will exclude you from the experiment and from any payment.

In the first phase of the experiment you will have to perform an individual task. You will have to memorize at least one verse of a poem, and report it correctly on a sheet of paper that will be provided by the assistants. You can keep the text of the poem for a minute, during which you will have to try to memorize the verse. At the end of this period, the assistant will collect the text of the poem, and give you a blank sheet of paper and a pen, which you can keep for another minute during which you can write the verse memorized. At the end of this period the assistant will collect the paper and check the correspondence with the text of the poem. 
If you report correctly at least one verse, you will get a prize of 3 euro. This sum of money will be paid at the end of the experiment together with the earnings of Phase 2.

Note that:

- The verse must be reported correctly.

- If it is not reported correctly or not reported at all, you will not earn anything.

- It is possible to write more than one verse, to be safe, but the verses in excess will not earn you any extra cash. Are there any questions?

[In the Ingroup and In-Complex conditions the text from the second paragraph was modified as follows:

At the beginning of the experiment you have been assigned a coloured bracelet. Players with red bracelets belong to the Red group, while those with the blue bracelet belong to the Blue group.

In the first phase of the experiment you will have to perform a collective task. The group will be asked to memorize a number of verses of a poem, and report them correctly on a sheet of paper that will be provided by the assistant. You can keep the text of the poem for five minutes, during which you will have to try to memorize the verses. At the end of this period, the assistant will collect the text, and give you a blank sheet of paper and a marker, which you can keep for another five minutes, during which you can write the verses you have memorized. At the end of this period the assistant will collect the paper and check the correspondence with the text of the poem.

If you report correctly at least 16 verses, the group will earn a prize of 48 euro to be divided equally among the members ( 3 euro each). The money will be paid at the end of the experiment together with the earnings of Phase 2 .

Note that:

- The verses must be reported correctly.

- If the correct verses are 15 or less, the group will fail and will not earn anything.

- It is possible to write more than 16 verses, to be safe, but the verses in excess will not earn you any extra cash. 
Are there any questions?]

\subsection{Stage 2}

The second phase of the experiment begins now. The instructions which we have distributed are only for your personal use. During this phase it is prohibited to communicate with the other participants. If you have any doubts or questions please ask the assistants. If you do not follow this rule, we will have to exclude you from the experiment and from any payment.

During the second phase of the experiment the participants who picked an odd number will have the opportunity to make choices that will affect your earnings. The participants with an even number instead will wait and will be remunerated according to the decisions made by the other participants. Overall, the experiment consists of 44 periods of play, and each period corresponds to a choice. The choices made by each subject will not be disclosed to other participants. The anonymity will be maintained both during and after the experiment: all the money you will earn will be paid privately at the end of the experiment.

\subsubsection{Description of decision}

During the experiment, each subject will play with another individual selected at random from the other participants [and identified only by a colour (red or blue, depending on the bracelet he/she is wearing) that identifies the group membership of each experimental subject.]

If you have picked an even number, you will not have to make any decision, but you will simply wait while the other participants make their decisions. If you have picked an odd number, you will have to make a series of 44 decisions that will determine your income and that of another experimental subject with an even number. Every decision is a choice between two [four] allocations of money, representing a profile of sums assigned by the decision maker to himself/herself and to another person selected at random among the

participants (called the recipient). The allocation chosen by the decision maker cannot be challenged by the recipient, who will play therefore an entirely passive role.

For example, during a round of play, a decision maker might be asked to choose between the following allocations, expressed in cents of a euro: 


\begin{tabular}{c|cc}
\hline & You & Other player \\
\hline$(1)$ & 1200 & 800 \\
$(2)$ & 800 & 1200 \\
\hline
\end{tabular}

If the decision maker chooses allocation (1) he/she earns 12 euro, and the recipient earns 8 euro. If the decision maker chooses (2) he/she earns 8 euro, and the recipient earns 12 euro.

[In the Complex and In-Complex conditions, the above options were replaced by the following ones:

\begin{tabular}{c|cc}
\hline & You & Other player \\
\hline$(1)$ & 1200 & 800 \\
$(2)$ & 600 & 1000 \\
$(3)$ & 800 & 1200 \\
$(4)$ & 1000 & 600 \\
\hline
\end{tabular}

If the decision maker chooses allocation (1) he/she earns 12 euro, and the recipient earns 8 euro. If the decision maker chooses (2) he/she earns 6 euro, and the recipient earns 10 euro. If the decision maker chooses (3) he/she earns 8 euro, and the recipient earns 12 euro. If he/she chooses (4), the decision maker earns 10 euro, and the recipient earns 6 euro.]

During and after the game the identity of the recipient will remain anonymous [and each player will be identified only by the colour of his/her own group (Red or Blue)]. Your decisions will be disclosed only to the recipient with whom you will be paired, which however will not be aware of your identity.

At the end of the experiment we will ask you to fill a short questionnaire. When you have finished, the assistants will draw randomly one of the 44 periods of play, and announce publicly the monetary allocations among which the decision-makers faced in that round. This way, each recipient will know which options were available to the decision-makers at the time of play.

The decision-makers will be called individually to the assistants desk where they will receive the amount of cash they have chosen for themselves in that period of the game, plus 
the money they have earned in the first phase of the experiment. Each payment will take place in private.

The money assigned to the recipient will then be placed into an anonymous envelope marked by a number [and by a colour (red or blue)] corresponding to that of the recipient. The assistant will draw randomly one of the even numbers and mark the envelope accordingly. When all the decision-makers will have been paid, each receiver will be called individually to the desk, where he/she will receive the envelope marked with his/her number. The envelope will contain the money awarded by the decision maker, plus the money earned in the first phase of the experiment, and will be handed to the recipient privately.

You will be asked to sign a receipt, and then you will be allowed to leave the room quietly. We will be grateful if you will not discuss the experiment with other students after leaving the laboratory. 